\title{
On the asymptotic expansions of products relating to the Wallis, Weierstrass, and Wilf formulas
}

Chao-Ping Chen, and Richard B. Paris

This is the accepted manuscript (c) 2016, Elsevier Licensed under the Creative Commons AttributionNonCommercial-NoDerivatives 4.0 International: http:// creativecommons.org/licenses/by-nc-nd/4.0/

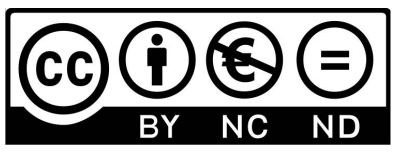

The published article is available from doi: https://dx.doi.org/10.1016/j.amc.2016.08.003 


\title{
On the asymptotic expansions of products related to the Wallis, Weierstrass, and Wilf formulas
}

\author{
Chao-Ping Chen ${ }^{a *}$ and Richard B. Paris ${ }^{b}$ \\ ${ }^{a}$ School of Mathematics and Informatics, Henan Polytechnic University, \\ Jiaozuo City 454000, Henan Province, China \\ Email: chenchaoping@sohu.com \\ ${ }^{b}$ Division of Computing and Mathematics, \\ University of Abertay, Dundee, DD1 1HG, UK \\ Email: R.Paris@abertay.ac.uk
}

Abstract: For all integers $n \geq 1$, let

$$
W_{n}(p, q)=\prod_{j=1}^{n}\left\{e^{-p / j}\left(1+\frac{p}{j}+\frac{q}{j^{2}}\right)\right\}
$$

and

$$
R_{n}(p, q)=\prod_{j=1}^{n}\left\{e^{-p /(2 j-1)}\left(1+\frac{p}{2 j-1}+\frac{q}{(2 j-1)^{2}}\right)\right\},
$$

where $p, q$ are complex parameters. The infinite product $W_{\infty}(p, q)$ includes the Wallis and Wilf formulas, and also the infinite product definition of Weierstrass for the gamma function, as special cases. In this paper, we present asymptotic expansions of $W_{n}(p, q)$ and $R_{n}(p, q)$ as $n \rightarrow \infty$. In addition, we also establish asymptotic expansions for the Wallis sequence.

2010 Mathematics Subject Classification: 33B15, 41A60

Keywords: Gamma function, Psi function, Euler-Mascheroni constant, Asymptotic expansion, Wallis sequence

\section{Introduction}

The famous Wallis sequence $W_{n}$, defined by

$$
W_{n}=\prod_{k=1}^{n} \frac{4 k^{2}}{4 k^{2}-1} \quad(n \in \mathbb{N}:=\{1,2,3, \ldots\})
$$

${ }^{*}$ Corresponding Author. 
has the limiting value

$$
W_{\infty}=\prod_{k=1}^{\infty} \frac{4 k^{2}}{4 k^{2}-1}=\frac{\pi}{2}
$$

established by Wallis in 1655; see [5, p. 68]. Several elementary proofs of this well-known result can be found in [3,23,37]. An interesting geometric construction that produces the above limiting value can be found in [30]. Many formulas exist for the representation of $\pi$, and a collection of these formulas is listed [33, 34]. For more history of $\pi$ see [2, 4, 5, 14].

The following infinite product definition for the gamma function is due to Weierstrass (see, for example, [1, p. 255, Entry (6.1.3)]):

$$
\frac{1}{\Gamma(z)}=z e^{\gamma z} \prod_{n=1}^{\infty}\left\{e^{-z / n}\left(1+\frac{z}{n}\right)\right\}
$$

where $\gamma$ denotes the Euler-Mascheroni constant defined by

$$
\gamma:=\lim _{n \rightarrow \infty}\left(\sum_{k=1}^{n} \frac{1}{k}-\ln n\right)=0.5772156649 \ldots
$$

In 1997, Wilf [39] posed the following elegant infinite product formula as a problem:

$$
\prod_{j=1}^{\infty}\left\{e^{-1 / j}\left(1+\frac{1}{j}+\frac{1}{2 j^{2}}\right)\right\}=\frac{e^{\pi / 2}+e^{-\pi / 2}}{\pi e^{\gamma}}
$$

which contains three of the most important mathematical constants, namely $\pi, e$ and $\gamma$. Subsequently, Choi and Seo [12] proved (1.4), together with three other similar product formulas, by making use of well-known infinite product formulas for the circular and hyperbolic functions and the familiar Stirling formula for the factorial function.

In 2003, Choi et al. [11] presented the following two general infinite product formulas, which include Wilf's formula (1.4) and other similar formulas in [12] as special cases:

$$
\prod_{j=1}^{\infty}\left\{e^{-1 / j}\left(1+\frac{1}{j}+\frac{\alpha^{2}+1 / 4}{j^{2}}\right)\right\}=\frac{2\left(e^{\pi \alpha}+e^{-\pi \alpha}\right)}{\left(4 \alpha^{2}+1\right) \pi e^{\gamma}} \quad\left(\alpha \in \mathbb{C} ; \alpha \neq \pm \frac{1}{2} i\right)
$$

and

$$
\prod_{j=1}^{\infty}\left\{e^{-2 / j}\left(1+\frac{2}{j}+\frac{\beta^{2}+1}{j^{2}}\right)\right\}=\frac{e^{\pi \beta}-e^{-\pi \beta}}{2 \beta\left(\beta^{2}+1\right) \pi e^{2 \gamma}} \quad(\beta \in \mathbb{C} \backslash\{0\} ; \beta \neq \pm i),
$$

where $i=\sqrt{-1}$ and $\mathbb{C}$ denotes the set of complex numbers. In 2013, Chen and Choi [7] presented a more general infinite product formula that included the formulas (1.5) and (1.6) as special cases:

$$
\prod_{j=1}^{\infty}\left\{e^{-p / j}\left(1+\frac{p}{j}+\frac{q}{j^{2}}\right)\right\}=\frac{e^{-p \gamma}}{\Gamma\left(1+\frac{1}{2} p+\frac{1}{2} \Delta\right) \Gamma\left(1+\frac{1}{2} p-\frac{1}{2} \Delta\right)}
$$


and also another interesting infinite product formula:

$$
\prod_{j=1}^{\infty}\left\{e^{-p /(2 j-1)}\left(1+\frac{p}{2 j-1}+\frac{q}{(2 j-1)^{2}}\right)\right\}=\frac{2^{-p} \pi e^{-p \gamma / 2}}{\Gamma\left(\frac{1}{2}+\frac{1}{4} p+\frac{1}{4} \Delta\right) \Gamma\left(\frac{1}{2}+\frac{1}{4} p-\frac{1}{4} \Delta\right)},
$$

where $p, q \in \mathbb{C}$ and $\Delta:=\sqrt{p^{2}-4 q}$.

The formula (1.7) can be seen to include the formulas (1.2)-(1.6) as special cases. By setting $(p, q)=$ $(0,-1 / 4)$ in 1.7$)$, we have

$$
\prod_{j=1}^{\infty}\left(1-\frac{1}{4 j^{2}}\right)=\frac{2}{\pi}
$$

whose reciprocal becomes the Wallis product (1.2). Also setting $q=0$ in 1.77, we obtain

$$
\prod_{j=1}^{\infty}\left\{e^{-p / j}\left(1+\frac{p}{j}\right)\right\}=\frac{e^{-p \gamma}}{\Gamma(p+1)}
$$

Noting that $\Gamma(z+1)=z \Gamma(z)$ and replacing $p$ by $z$ in (1.10) we recover the Weierstrass formula (1.3). Setting $(p, q)=(1,1 / 2)$ in (1.7) yields the Wilf formula (1.4) and setting

$$
(p, q)=\left(1, \alpha^{2}+\frac{1}{4}\right) \quad \text { and } \quad(p, q)=\left(2, \beta^{2}+1\right)
$$

in (1.7) yields the formulas (1.5) and (1.6), respectively.

With $(p, q)=(-1,1 / 4)$ in 1.7 , we obtain the beautiful infinite product formula expressed in terms of the most important constants $\pi, e$ and $\gamma$, namely

$$
\prod_{j=1}^{\infty}\left\{e^{1 / j}\left(1-\frac{1}{2 j}\right)^{2}\right\}=\frac{e^{\gamma}}{\pi}
$$

Also worthy of note are the infinite products that result from setting $(p, q)=(-2,0)$ and $(p, q)=(2,0)$ in (1.8) to yield respectively

$$
\prod_{j=1}^{\infty}\left\{e^{2 /(2 j-1)}\left(1-\frac{2}{2 j-1}\right)\right\}=-2 e^{\gamma}
$$

and

$$
\prod_{j=1}^{\infty}\left\{e^{-2 /(2 j-1)}\left(1+\frac{2}{2 j-1}\right)\right\}=\frac{1}{2 e^{\gamma}} .
$$

Remark 1.1. The constant $e^{\gamma}$ is important in number theory and equals the following limit, where $p_{n}$ is the nth prime number:

$$
e^{\gamma}=\lim _{n \rightarrow \infty} \frac{1}{\ln p_{n}} \prod_{j=1}^{n} \frac{p_{j}}{p_{j}-1} .
$$

This restates the third of Mertens' theorems (see [38]). The numerical value of $e^{\gamma}$ is:

$$
e^{\gamma}=1.7810724179 \ldots
$$


There is the curious radical representation

$$
e^{\gamma}=\left(\frac{2}{1}\right)^{1 / 2}\left(\frac{2^{2}}{1 \cdot 3}\right)^{1 / 3}\left(\frac{2^{3} \cdot 4}{1 \cdot 3^{3}}\right)^{1 / 4}\left(\frac{2^{4} \cdot 4^{4}}{1 \cdot 3^{6} \cdot 5}\right)^{1 / 5} \cdots,
$$

where the nth factor is

$$
\left(\prod_{k=0}^{n}(k+1)^{(-1)^{k+1}\left(\begin{array}{c}
n \\
k
\end{array}\right)}\right)^{1 /(n+1)} .
$$

The product [1.15], first discovered in 1926 by Ser [32], was rediscovered in [16] 35] 36].

Recently, Chen and Paris [9] generalized the formula (1.7) to include $m$ parameters $\left(p_{1}, \ldots, p_{m}\right)$. Subsequently, Chen and Paris [10] considered the asymptotic expansion of products related to generalization of the Wilf problem. However, these authors did not give a general formula for the coefficients in their expansions.

For $n \in \mathbb{N}$, let

$$
W_{n}(p, q)=\prod_{j=1}^{n}\left\{e^{-p / j}\left(1+\frac{p}{j}+\frac{q}{j^{2}}\right)\right\}
$$

and

$$
R_{n}(p, q)=\prod_{j=1}^{n}\left\{e^{-p /(2 j-1)}\left(1+\frac{p}{2 j-1}+\frac{q}{(2 j-1)^{2}}\right)\right\},
$$

where $p$ and $q$ are complex parameters. In this paper, we present asymptotic expansions of $W_{n}(p, q)$ and $R_{n}(p, q)$ as $n \rightarrow \infty$, including recurrence relations for the coefficients in these expansions. Furthermore, we establish asymptotic expansions for the Wallis sequence $W_{n}$.

\section{Asymptotic expansions of $W_{n}(p, q)$ and $R_{n}(r, s)$}

It was established by Chen and Choi [7] that the finite products $W_{n}(p, q)$ and $R_{n}(p, q)$ defined in 1.16) and 1.17 can be expressed in the following closed form

$$
W_{n}(p, q)=\frac{e^{-p(\psi(n+1)+\gamma)} \Gamma\left(n+1+\frac{1}{2} p+\frac{1}{2} \Delta\right) \Gamma\left(n+1+\frac{1}{2} p-\frac{1}{2} \Delta\right)}{(\Gamma(n+1))^{2} \Gamma\left(1+\frac{1}{2} p+\frac{1}{2} \Delta\right) \Gamma\left(1+\frac{1}{2} p-\frac{1}{2} \Delta\right)}
$$

and

$$
R_{n}(p, q)=\frac{e^{-\frac{p}{2}\left(\psi\left(n+\frac{1}{2}\right)+\gamma+2 \ln 2\right)} \Gamma\left(n+\frac{1}{2}+\frac{1}{4} p+\frac{1}{4} \Delta\right) \Gamma\left(n+\frac{1}{2}+\frac{1}{4} p-\frac{1}{4} \Delta\right) \pi}{\left(\Gamma\left(n+\frac{1}{2}\right)\right)^{2} \Gamma\left(\frac{1}{2}+\frac{1}{4} p+\frac{1}{4} \Delta\right) \Gamma\left(\frac{1}{2}+\frac{1}{4} p-\frac{1}{4} \Delta\right)},
$$

where $\psi(z)$ denotes the psi (or digamma) function, defined by

$$
\psi(z)=\frac{\mathrm{d}}{\mathrm{d} z}\{\ln \Gamma(z)\}=\frac{\Gamma^{\prime}(z)}{\Gamma(z)} .
$$

We observe that allowing $n \rightarrow \infty$ in (2.1) and (2.2), respectively, yields (1.7) and (1.8). 
Define the function $f(z)$ by

$$
f(z):=\frac{e^{-\lambda \psi(z)} \Gamma(z+\mu) \Gamma(z+\nu)}{(\Gamma(z))^{2}},
$$

where $\lambda, \mu, \nu \in \mathbb{C}$. It is well known that the logarithm of the gamma function has the asymptotic expansion (see [22, p. 32]):

$$
\ln \Gamma(z+a) \sim\left(z+a-\frac{1}{2}\right) \ln z-z+\frac{1}{2} \ln (2 \pi)+\sum_{n=1}^{\infty} \frac{(-1)^{n+1} B_{n+1}(a)}{n(n+1)} \frac{1}{z^{n}}
$$

for $z \rightarrow \infty$ in $|\arg z|<\pi$, where $B_{n}(t)$ denote the Bernoulli polynomials defined by the following generating function:

$$
\frac{z e^{t z}}{e^{z}-1}=\sum_{n=0}^{\infty} B_{n}(t) \frac{z^{n}}{n !}
$$

Note that the Bernoulli numbers $B_{n}\left(n \in \mathbb{N}_{0}:=\mathbb{N} \cup\{0\}\right)$ are defined by $B_{n}:=B_{n}(0)$. The psi function has the asymptotic expansion (see [22, p. 33]):

$$
\psi(z) \sim \ln z-\frac{1}{z}-\sum_{j=1}^{\infty} \frac{B_{j}}{j z^{j}} \quad(z \rightarrow \infty ;|\arg z|<\pi) .
$$

Using (2.4) and (2.5), we then find that

$$
\ln f(z) \sim(\mu+\nu-\lambda) \ln z+\sum_{j=1}^{\infty} \frac{a_{j}}{z^{j}}
$$

or

$$
f(z) \sim z^{\mu+\nu-\lambda} \exp \left(\sum_{j=1}^{\infty} \frac{a_{j}}{z^{j}}\right)
$$

for $z \rightarrow \infty$ in $|\arg z|<\pi$, where the coefficients $a_{j} \equiv a_{j}(\lambda, \mu, \nu)$ are given by

$$
a_{1}=\frac{\lambda+B_{2}(\mu)+B_{2}(\nu)-2 B_{2}}{2}, \quad a_{j}=\frac{\lambda B_{j}}{j}+\frac{(-1)^{j+1}\left(B_{j+1}(\mu)+B_{j+1}(\nu)-2 B_{j+1}\right)}{j(j+1)} \quad(j \geq 2) \text {. }
$$

The choice

$$
(\lambda, \mu, \nu)=\left(p, \frac{1}{2} p+\frac{1}{2} \Delta, \frac{1}{2} p-\frac{1}{2} \Delta\right),
$$

where $\mu+\nu-\lambda=0$, leads to the first few coefficients $a_{j}(p, q)$ given by:

$$
\begin{aligned}
& a_{1}(p, q)=\frac{1}{2} p^{2}-q \\
& a_{2}(p, q)=-\frac{1}{6} p^{3}+\frac{1}{2} p q+\frac{1}{4} p^{2}-\frac{1}{2} q \\
& a_{3}(p, q)=\frac{1}{12} p^{4}-\frac{1}{3} p^{2} q+\frac{1}{6} q^{2}-\frac{1}{6} p^{3}+\frac{1}{2} p q+\frac{1}{12} p^{2}-\frac{1}{6} q .
\end{aligned}
$$

From (2.1) and 2.6, we obtain the following 
Theorem 2.1. As $n \rightarrow \infty$, we have

$$
W_{n}(p, q) \sim \frac{e^{-p \gamma}}{\Gamma\left(1+\frac{1}{2} p+\frac{1}{2} \Delta\right) \Gamma\left(1+\frac{1}{2} p-\frac{1}{2} \Delta\right)} \exp \left(\sum_{j=1}^{\infty} \frac{a_{j}(p, q)}{(n+1)^{j}}\right),
$$

where the coefficients $a_{j}(p, q)$ are given by

$$
\begin{aligned}
& a_{1}(p, q)=\frac{1}{2} p^{2}-q \quad \text { and } \\
& a_{j}(p, q)=\frac{p B_{j}}{j}+\frac{(-1)^{j+1}\left(B_{j+1}\left(\frac{1}{2} p+\frac{1}{2} \Delta\right)+B_{j+1}\left(\frac{1}{2} p-\frac{1}{2} \Delta\right)-2 B_{j+1}\right)}{j(j+1)} \quad(j \geq 2) .
\end{aligned}
$$

Thus we have the expansion

$$
\begin{aligned}
W_{n}(p, q) \sim & \frac{e^{-p \gamma}}{\Gamma\left(1+\frac{1}{2} p+\frac{1}{2} \Delta\right) \Gamma\left(1+\frac{1}{2} p-\frac{1}{2} \Delta\right)} \\
& \times \exp \left(\frac{\frac{1}{2} p^{2}-q}{n+1}+\frac{-\frac{1}{6} p^{3}+\frac{1}{2} p q+\frac{1}{4} p^{2}-\frac{1}{2} q}{(n+1)^{2}}\right. \\
& \left.+\frac{\frac{1}{12} p^{4}-\frac{1}{3} p^{2} q+\frac{1}{6} q^{2}-\frac{1}{6} p^{3}+\frac{1}{2} p q+\frac{1}{12} p^{2}-\frac{1}{6} q}{(n+1)^{3}}+\cdots\right)
\end{aligned}
$$

as $n \rightarrow \infty$.

Remark 2.1 Note that since $W_{n}=1 / W_{n}\left(0,-\frac{1}{4}\right)$, it follows by setting $(p, q)=\left(0,-\frac{1}{4}\right)$ in (2.10) that

$$
W_{n} \sim \frac{\pi}{2} \exp \left(-\frac{1}{4(n+1)}-\frac{1}{8(n+1)^{2}}-\frac{5}{96(n+1)^{3}}-\cdots\right)
$$

as $n \rightarrow \infty$.

The same procedure with the choice

$$
(\lambda, \mu, \nu)=\left(\frac{1}{2} p, \frac{1}{4} p+\frac{1}{4} \Delta, \frac{1}{4} p+\frac{1}{4} \Delta\right)
$$

in (2.2) and 2.6) leads to the following

Theorem 2.2.. As $n \rightarrow \infty$, we have

$$
R_{n}(p, q) \sim \frac{2^{-p} \pi e^{-p \gamma / 2}}{\Gamma\left(\frac{1}{2}+\frac{1}{4} p+\frac{1}{4} \Delta\right) \Gamma\left(\frac{1}{2}+\frac{1}{4} p-\frac{1}{4} \Delta\right)} \exp \left(\sum_{j=1}^{\infty} \frac{b_{j}(p, q)}{\left(n+\frac{1}{2}\right)^{j}}\right),
$$

where the coefficients $b_{j}(p, q)$ are given by

$$
\begin{aligned}
& b_{1}(r, s)=\frac{1}{8} p^{2}-\frac{1}{4} q \text { and } \\
& b_{j}(r, s)=\frac{p B_{j}}{2 j}+\frac{(-1)^{j+1}\left(B_{j+1}\left(\frac{1}{4} p+\frac{1}{4} \Delta\right)+B_{j+1}\left(\frac{1}{4} p-\frac{1}{4} \Delta\right)-2 B_{j+1}\right)}{j(j+1)} \quad(j \geq 2) .
\end{aligned}
$$


Thus we have the expansion

$$
\begin{aligned}
R_{n}(p, q) \sim & \frac{2^{-p} \pi e^{-p \gamma / 2}}{\Gamma\left(\frac{1}{2}+\frac{1}{4} p+\frac{1}{4} \Delta\right) \Gamma\left(\frac{1}{2}+\frac{1}{4} p-\frac{1}{4} \Delta\right)} \\
& \times \exp \left(\frac{\frac{1}{8} p^{2}-\frac{1}{4} q}{n+\frac{1}{2}}+\frac{-\frac{1}{48} p^{3}+\frac{1}{16} p q+\frac{1}{16} p^{2}-\frac{1}{8} q}{\left(n+\frac{1}{2}\right)^{2}}\right. \\
& \left.+\frac{\frac{1}{192} p^{4}-\frac{1}{48} p^{2} q+\frac{1}{96} q^{2}-\frac{1}{48} p^{3}+\frac{1}{16} p q+\frac{1}{48} p^{2}-\frac{1}{24} q}{\left(n+\frac{1}{2}\right)^{3}}+\cdots\right)
\end{aligned}
$$

as $n \rightarrow \infty$.

The first two terms in the expansions (2.10) and 2.14 can be shown to agree with the expansions in inverse powers of $n$ obtained in [10, Eqs. (4.3), (4.4)].

\section{Asymptotic series expansions of the Wallis sequence}

Some inequalities and asymptotic formulas associated with the Wallis sequence $W_{n}$ can be found in [6, 13, 15, 17,-21, 24]-29, 31]. For example, Elezović et al. [15] showed that the following asymptotic expansion holds:

$$
W_{n} \sim \frac{\pi}{2}\left(1-\frac{\frac{1}{4}}{n+\frac{5}{8}}+\frac{\frac{3}{256}}{\left(n+\frac{5}{8}\right)^{3}}+\frac{\frac{3}{2048}}{\left(n+\frac{5}{8}\right)^{4}}-\frac{\frac{51}{16384}}{\left(n+\frac{5}{8}\right)^{5}}-\frac{\frac{75}{65536}}{\left(n+\frac{5}{8}\right)^{6}}+\frac{\frac{2253}{1048576}}{\left(n+\frac{5}{8}\right)^{7}}+\cdots\right)
$$

as $n \rightarrow \infty$. Deng et al. [13] proved that for all $n \in \mathbb{N}$,

$$
\frac{\pi}{2}\left(1-\frac{1}{4 n+\alpha}\right)<W_{n} \leq \frac{\pi}{2}\left(1-\frac{1}{4 n+\beta}\right)
$$

with the best possible constants

$$
\alpha=\frac{5}{2} \quad \text { and } \quad \beta=\frac{32-9 \pi}{3 \pi-8}=2.614909986 \ldots
$$

In fact, Elezović et al. [15] have previously shown that $\frac{5}{2}$ is the best possible constant for a lower bound of $W_{n}$ of the type $\frac{\pi}{2}\left(1-\frac{1}{4 n+\alpha}\right)$. Moreover, the authors pointed out that

$$
W_{n}=\frac{\pi}{2}\left(1-\frac{1}{4 n+\frac{5}{2}}\right)+O\left(\frac{1}{n^{3}}\right) \quad(n \rightarrow \infty) .
$$

Here, we will establish two more accurate asymptotic expansions for $W_{n}$ (see Theorems 3.1 and 3.2) by making use of the fact that

$$
W_{n}=\frac{\pi}{2} \cdot \frac{1}{n+\frac{1}{2}}\left[\frac{\Gamma(n+1)}{\Gamma\left(n+\frac{1}{2}\right)}\right]^{2}=\frac{\pi}{2} \cdot \frac{\Gamma(n+1)^{2}}{\Gamma\left(n+\frac{1}{2}\right) \Gamma\left(n+\frac{3}{2}\right)} .
$$

The following lemma is required in our present investigation. 
Lemma 3.1 (see [8]). Let

$$
A(x) \sim \sum_{n=1}^{\infty} a_{n} x^{-n} \quad(x \rightarrow \infty)
$$

be a given asymptotical expansion. Then the composition $\exp (A(x))$ has asymptotic expansion of the following form

$$
\exp (A(x)) \sim \sum_{n=0}^{\infty} b_{n} x^{-n} \quad(x \rightarrow \infty)
$$

where

$$
b_{0}=1, \quad b_{n}=\frac{1}{n} \sum_{k=1}^{n} k a_{k} b_{n-k} \quad(n \geq 1) .
$$

From (2.4), we find as $n \rightarrow \infty$

$$
W_{n} \sim \frac{\pi}{2} \exp \left(\sum_{j=1}^{\infty} \frac{\nu_{j}}{n^{j}}\right),
$$

where the coefficients $\nu_{j}$ are given by

$$
\nu_{j}=\frac{(-1)^{j+1}\left(2 B_{j+1}-B_{j+1}\left(\frac{1}{2}\right)-B_{j+1}\left(\frac{3}{2}\right)\right)}{j(j+1)} \quad(j \geq 1) .
$$

Noting that (see [1, pp. 805-804])

$$
B_{n}(1-x)=(-1)^{n} B_{n}(x), \quad(-1)^{n} B_{n}(-x)=B_{n}(x)+n x^{n-1} \quad\left(n \in \mathbb{N}_{0}\right)
$$

and

$$
B_{n}\left(\frac{1}{2}\right)=-\left(1-2^{1-n}\right) B_{n} \quad\left(n \in \mathbb{N}_{0}\right),
$$

we find that (3.7) can be written as

$$
\nu_{j}=\frac{(-1)^{j+1}\left(\left(4-2^{1-j}\right) B_{j+1}-(j+1) \cdot 2^{-j}\right)}{j(j+1)} \quad(j \geq 1) .
$$

Thus, we obtain the expansion

$$
\begin{aligned}
W_{n} \sim \frac{\pi}{2} \exp ( & -\frac{1}{4 n}+\frac{1}{8 n^{2}}-\frac{5}{96 n^{3}}+\frac{1}{64 n^{4}}-\frac{1}{320 n^{5}}+\frac{1}{384 n^{6}}-\frac{25}{7168 n^{7}} \\
& \left.+\frac{1}{2048 n^{8}}+\frac{29}{9216 n^{9}}+\frac{1}{10240 n^{10}}-\frac{695}{90112 n^{11}}+\cdots\right) .
\end{aligned}
$$


By Lemma 3.1, we then obtain from 3.6

$$
W_{n} \sim \frac{\pi}{2} \sum_{j=0}^{\infty} \frac{\mu_{j}}{n^{j}}
$$

where the coefficients $\mu_{j}$ are given by the recurrence relation

$$
\mu_{0}=1, \quad \mu_{j}=\frac{1}{j} \sum_{k=1}^{j} k \nu_{k} \mu_{j-k} \quad(j \geq 1) .
$$

and the $\nu_{j}$ are given in (3.8). This produces the expansion in inverse powers of $n$ given by

$$
\begin{aligned}
W_{n} \sim \frac{\pi}{2}( & -\frac{1}{4 n}+\frac{5}{32 n^{2}}-\frac{11}{128 n^{3}}+\frac{83}{2048 n^{4}}-\frac{143}{8192 n^{5}}+\frac{625}{65536 n^{6}}-\frac{1843}{262144 n^{7}} \\
& \left.+\frac{24323}{8388608 n^{8}}+\frac{61477}{33554432 n^{9}}-\frac{14165}{268435456 n^{10}}-\frac{8084893}{1073741824 n^{11}}+\cdots\right)
\end{aligned}
$$

as $n \rightarrow \infty$.

Theorem 3.1. The Wallis sequence has the following asymptotic expansion:

$$
W_{n} \sim \frac{\pi}{2}\left(1+\sum_{\ell=1}^{\infty} \frac{\alpha_{\ell}}{\left(n+\beta_{\ell}\right)^{2 \ell-1}}\right) \quad(n \rightarrow \infty),
$$

where $\alpha_{\ell}$ and $\beta_{\ell}$ are given by the pair of recurrence relations

$$
\alpha_{\ell}=\mu_{2 \ell-1}-\sum_{k=1}^{\ell-1} \alpha_{k} \beta_{k}^{2 \ell-2 k}\left(\begin{array}{c}
2 \ell-2 \\
2 \ell-2 k
\end{array}\right) \quad(\ell \geq 2)
$$

and

$$
\beta_{\ell}=-\frac{1}{(2 \ell-1) \alpha_{\ell}}\left\{\mu_{2 \ell}+\sum_{k=1}^{\ell-1} \alpha_{k} \beta_{k}^{2 \ell-2 k+1}\left(\begin{array}{c}
2 \ell-1 \\
2 \ell-2 k+1
\end{array}\right)\right\} \quad(\ell \geq 2)
$$

with $\alpha_{1}=-\frac{1}{4}$ and $\beta_{1}=\frac{5}{8}$. Here $\mu_{j}$ are given by the recurrence relation 3.11).

Proof. Let

$$
W_{n} \sim \frac{\pi}{2}\left(1+\sum_{\ell=1}^{\infty} \frac{\alpha_{\ell}}{\left(n+\beta_{\ell}\right)^{2 \ell-1}}\right) \quad(n \rightarrow \infty),
$$

where $\alpha_{\ell}$ and $\beta_{\ell}$ are real numbers to be determined. This can be written as

$$
\frac{2}{\pi} W_{n} \sim 1+\sum_{j=1}^{\infty} \frac{\alpha_{j}}{n^{2 j-1}}\left(1+\frac{\beta_{j}}{n}\right)^{-2 j+1} .
$$


Direct computation yields

$$
\begin{aligned}
\sum_{j=1}^{\infty} \frac{\alpha_{j}}{n^{2 j-1}}\left(1+\frac{\beta_{j}}{n}\right)^{-2 j+1} & \sim \sum_{j=1}^{\infty} \frac{\alpha_{j}}{n^{2 j-1}} \sum_{k=0}^{\infty}\left(\begin{array}{c}
-2 j+1 \\
k
\end{array}\right) \frac{\beta_{j}^{k}}{n^{k}} \\
& \sim \sum_{j=1}^{\infty} \frac{\alpha_{j}}{n^{2 j-1}} \sum_{k=0}^{\infty}(-1)^{k}\left(\begin{array}{c}
k+2 j-2 \\
k
\end{array}\right) \frac{\beta_{j}^{k}}{n^{k}} \\
& \sim \sum_{j=1}^{\infty} \sum_{k=0}^{j-1} \alpha_{k+1} \beta_{k+1}^{j-k-1}(-1)^{j-k-1}\left(\begin{array}{l}
j+k-1 \\
j-k-1
\end{array}\right) \frac{1}{n^{j+k}},
\end{aligned}
$$

which can be written as

$$
\sum_{j=1}^{\infty} \frac{\alpha_{j}}{n^{2 j-1}}\left(1+\frac{\beta_{j}}{n}\right)^{-2 j+1} \sim \sum_{j=1}^{\infty}\left\{\sum_{k=1}^{\left\lfloor\frac{j+1}{2}\right\rfloor} \alpha_{k} \beta_{k}^{j-2 k+1}(-1)^{j-1}\left(\begin{array}{c}
j-1 \\
j-2 k+1
\end{array}\right)\right\} \frac{1}{n^{j}} .
$$

It then follows from (3.16) and (3.17) that

$$
\frac{2}{\pi} W_{n} \sim 1+\sum_{j=1}^{\infty}\left\{\sum_{k=1}^{\left\lfloor\frac{j+1}{2}\right\rfloor} \alpha_{k} \beta_{k}^{j-2 k+1}(-1)^{j-1}\left(\begin{array}{c}
j-1 \\
j-2 k+1
\end{array}\right)\right\} \frac{1}{n^{j}} .
$$

On the other hand, we have from (3.10) that

$$
\frac{2}{\pi} W_{n} \sim 1+\sum_{j=1}^{\infty} \frac{\mu_{j}}{n^{j}} .
$$

Equating coefficients of $n^{-j}$ on the right-hand sides of (3.18) and (3.19), we obtain

$$
\mu_{j}=\sum_{k=1}^{\left\lfloor\frac{j+1}{2}\right\rfloor} \alpha_{k} \beta_{k}^{j-2 k+1}(-1)^{j-1}\left(\begin{array}{c}
j-1 \\
j-2 k+1
\end{array}\right) \quad(j \in \mathbb{N}) .
$$

Setting $j=2 \ell-1$ and $j=2 \ell$ in $(3.20)$, respectively, we find

$$
\mu_{2 \ell-1}=\sum_{k=1}^{\ell} \alpha_{k} \beta_{k}^{2 \ell-2 k}\left(\begin{array}{c}
2 \ell-2 \\
2 \ell-2 k
\end{array}\right)
$$

and

$$
\mu_{2 \ell}=-\sum_{k=1}^{\ell} \alpha_{k} \beta_{k}^{2 \ell-2 k+1}\left(\begin{array}{c}
2 \ell-1 \\
2 \ell-2 k+1
\end{array}\right) .
$$

For $\ell=1$, we obtain from (3.21) and (3.22)

$$
\alpha_{1}=\mu_{1}=-\frac{1}{4} \quad \text { and } \quad \beta_{1}=-\frac{\mu_{2}}{\alpha_{1}}=\frac{5}{8},
$$


and for $\ell \geq 2$ we have

$$
\mu_{2 \ell-1}=\sum_{k=1}^{\ell-1} \alpha_{k} \beta_{k}^{2 \ell-2 k}\left(\begin{array}{c}
2 \ell-2 \\
2 \ell-2 k
\end{array}\right)+\alpha_{\ell}
$$

and

$$
\mu_{2 \ell}=-\sum_{k=1}^{\ell-1} \alpha_{k} \beta_{k}^{2 \ell-2 k+1}\left(\begin{array}{c}
2 \ell-1 \\
2 \ell-2 k+1
\end{array}\right)-(2 \ell-1) \alpha_{\ell} \beta_{\ell} .
$$

We then obtain the recurrence relations (3.14) and 3.15). The proof of Theorem 3.1] is complete.

We now give explicit numerical values of the first few $\alpha_{\ell}$ and $\beta_{\ell}$ by using the recurrence relations (3.14) and (3.15). This demonstrates the ease with which the constants $\alpha_{\ell}$ and $\beta_{\ell}$ in (3) can be determined. We find

$$
\begin{aligned}
& \alpha_{1}=-\frac{1}{4}, \quad \beta_{1}=\frac{5}{8} \\
& \alpha_{2}=\mu_{3}-\alpha_{1} \beta_{1}^{2}=-\frac{11}{128}-\left(-\frac{1}{4}\right) \cdot\left(\frac{5}{8}\right)^{2}=\frac{3}{256} \\
& \beta_{2}=-\frac{\mu_{4}+\alpha_{1} \beta_{1}^{3}}{3 \alpha_{2}}=-\frac{\frac{83}{2048}+\left(-\frac{1}{4}\right) \cdot\left(\frac{5}{8}\right)^{3}}{3 \cdot\left(\frac{3}{256}\right)}=\frac{7}{12} \\
& \alpha_{3}=\mu_{5}-\alpha_{1} \beta_{1}^{4}-6 \alpha_{2} \beta_{2}^{2}=-\frac{143}{8192}-\left(-\frac{1}{4}\right) \cdot\left(\frac{5}{8}\right)^{4}-6 \cdot\left(\frac{3}{256}\right) \cdot\left(\frac{7}{12}\right)^{2}=-\frac{53}{16384} \\
& \beta_{3}=-\frac{\mu_{6}+\alpha_{1} \beta_{1}^{5}+10 \alpha_{2} \beta_{2}^{3}}{5 \alpha_{3}}=-\frac{\frac{625}{65536}+\left(-\frac{1}{4}\right) \cdot\left(\frac{5}{8}\right)^{5}+10 \cdot\left(\frac{3}{256}\right) \cdot\left(\frac{7}{12}\right)^{3}}{5 \cdot\left(-\frac{53}{16384}\right)}=\frac{2113}{3816}
\end{aligned}
$$

Continuation of this procedure then enables the following coefficients to be derived:

$$
\begin{aligned}
\alpha_{4} & =\frac{224573}{93782016}, \quad \beta_{4}=\frac{22119189899}{41134587264}, \\
\alpha_{5} & =-\frac{596297240983745796931}{176651089583152098705408}, \quad \beta_{5}=\frac{38909478384301921254232134966821}{73585322683584986068354328660352} .
\end{aligned}
$$

We then obtain the following explicit asymptotic expansion:

$$
\begin{aligned}
W_{n} \sim \frac{\pi}{2}(1 & -\frac{\frac{1}{4}}{n+\frac{5}{8}}+\frac{\frac{3}{256}}{\left(n+\frac{7}{12}\right)^{3}}-\frac{\frac{53}{16384}}{\left(n+\frac{2113}{3816}\right)^{5}} \\
& \left.+\frac{\frac{224573}{93782016}}{\left(n+\frac{22119189899}{41134587264}\right)^{7}}-\frac{\frac{59629724098837457969631}{176651089583152008705408}}{\left(n+\frac{38909478384301921254232134966821}{73585322683584986068354328660352}\right)^{9}}+\cdots\right) .
\end{aligned}
$$

Thus, we would appear to obtain an alternating odd-type asymptotic expansion for $W_{n}$. From a computational viewpoint, (3.23) is an improvement on the formulas (3.12) and 3.1].

Theorem 3.2. The Wallis sequence has the following asymptotic expansion:

$$
W_{n} \sim \frac{\pi}{2} \exp \left(\sum_{\ell=1}^{\infty} \frac{\omega_{\ell}}{\left(n+\frac{1}{2}\right)^{2 \ell-1}}\right) \quad(n \rightarrow \infty)
$$


with the coefficients $\omega_{\ell}$ given by the recurrence relation

$$
\omega_{1}=-\frac{1}{4} \quad \text { and } \quad \omega_{\ell}=\nu_{2 \ell-1}-\sum_{k=1}^{\ell-1} \omega_{k}\left(\frac{1}{2}\right)^{2 \ell-2 k}\left(\begin{array}{c}
2 \ell-2 \\
2 \ell-2 k
\end{array}\right) \quad(\ell \geq 2),
$$

where the $\nu_{j}$ are given in (3.8).

Proof. Let

$$
W_{n} \sim \frac{\pi}{2} \exp \left(\sum_{\ell=1}^{\infty} \frac{\omega_{\ell}}{\left(n+\frac{1}{2}\right)^{2 \ell-1}}\right) \quad(n \rightarrow \infty),
$$

where $\omega_{\ell}$ are real numbers to be determined. This can be written as

$$
\ln \left(\frac{2}{\pi} W_{n}\right) \sim \sum_{j=1}^{\infty} \frac{\omega_{j}}{n^{2 j-1}}\left(1+\frac{1}{2 n}\right)^{-2 j+1} .
$$

The choice $\beta_{j}=\frac{1}{2}$ in 3.17 , with $\alpha_{j}$ replaced by $\omega_{j}$, yields

$$
\sum_{j=1}^{\infty} \frac{\omega_{j}}{n^{2 j-1}}\left(1+\frac{1}{2 n}\right)^{-2 j+1} \sim \sum_{j=1}^{\infty}\left\{\sum_{k=1}^{\left\lfloor\frac{j+1}{2}\right\rfloor} \omega_{k}\left(\frac{1}{2}\right)^{j-2 k+1}(-1)^{j-1}\left(\begin{array}{c}
j-1 \\
j-2 k+1
\end{array}\right)\right\} \frac{1}{n^{j}} .
$$

We then obtain

$$
\ln \left(\frac{2}{\pi} W_{n}\right) \sim \sum_{j=1}^{\infty}\left\{\sum_{k=1}^{\left\lfloor\frac{j+1}{2}\right\rfloor} \omega_{k}\left(\frac{1}{2}\right)^{j-2 k+1}(-1)^{j-1}\left(\begin{array}{c}
j-1 \\
j-2 k+1
\end{array}\right)\right\} \frac{1}{n^{j}} .
$$

On the other hand, we have from (3.6) that

$$
\ln \left(\frac{2}{\pi} W_{n}\right) \sim \sum_{j=1}^{\infty} \frac{\nu_{j}}{n^{j}} .
$$

Equating coefficients of $n^{-j}$ on the right-hand sides of 3.26) and 3.27, we obtain

$$
\nu_{j}=\sum_{k=1}^{\left\lfloor\frac{j+1}{2}\right\rfloor} \omega_{k}\left(\frac{1}{2}\right)^{j-2 k+1}(-1)^{j-1}\left(\begin{array}{c}
j-1 \\
j-2 k+1
\end{array}\right) \quad(j \in \mathbb{N}) .
$$

Setting $j=2 \ell-1$ in $(3.28)$, we find

$$
\nu_{2 \ell-1}=\sum_{k=1}^{\ell} \omega_{k}\left(\frac{1}{2}\right)^{2 \ell-2 k}\left(\begin{array}{c}
2 \ell-2 \\
2 \ell-2 k
\end{array}\right) .
$$

Substitution of $\ell=1$ in (3.29) yields $\omega_{1}=\nu_{1}=-\frac{1}{4}$, and for $\ell \geq 2$ we have

$$
\nu_{2 \ell-1}=\sum_{k=1}^{\ell-1} \omega_{k}\left(\frac{1}{2}\right)^{2 \ell-2 k}\left(\begin{array}{c}
2 \ell-2 \\
2 \ell-2 k
\end{array}\right)+\omega_{\ell} .
$$

We then obtain the recurrence relation (3.25). The proof of Theorem 3.2 is complete. 
Remark 3.1. Setting $j=2 \ell$ in 3.28 , we find

$$
\nu_{2 \ell}=-\sum_{k=1}^{\ell} \omega_{k}\left(\frac{1}{2}\right)^{2 \ell-2 k+1}\left(\begin{array}{c}
2 \ell-1 \\
2 \ell-2 k+1
\end{array}\right) .
$$

For $\ell=1$ in (3.30) this yields $\omega_{1}=-2 \nu_{2}=-\frac{1}{4}$, and for $\ell \geq 2$ we have

$$
\nu_{2 \ell}=-\sum_{k=1}^{\ell-1} \omega_{k}\left(\frac{1}{2}\right)^{2 \ell-2 k+1}\left(\begin{array}{c}
2 \ell-1 \\
2 \ell-2 k+1
\end{array}\right)-\left(\ell-\frac{1}{2}\right) \omega_{\ell} .
$$

We then obtain the alternative recurrence relation for the coefficients $\omega_{j}$ in (3.24) in terms of the even coefficients $\nu_{j}$ :

$$
\omega_{1}=-\frac{1}{4} \quad \text { and } \quad \omega_{\ell}=-\frac{2}{2 \ell-1}\left\{\nu_{2 \ell}+\sum_{k=1}^{\ell-1} \omega_{k}\left(\frac{1}{2}\right)^{2 \ell-2 k+1}\left(\begin{array}{c}
2 \ell-1 \\
2 \ell-2 k+1
\end{array}\right)\right\} \quad(\ell \geq 2) .
$$

Hence, from 3.24, we obtain the following explicit asymptotic expansion:

$$
W_{n} \sim \frac{\pi}{2} \exp \left(-\frac{\frac{1}{4}}{n+\frac{1}{2}}+\frac{\frac{1}{96}}{\left(n+\frac{1}{2}\right)^{3}}-\frac{\frac{1}{320}}{\left(n+\frac{1}{2}\right)^{5}}+\frac{\frac{17}{7168}}{\left(n+\frac{1}{2}\right)^{7}}-\frac{\frac{31}{9216}}{\left(n+\frac{1}{2}\right)^{9}}+\cdots\right) .
$$

This would appear to be an alternating odd-type expansion for $W_{n}$. From a computational viewpoint, (3.32) is an improvement on the formulas (2.11) and (3.9).

\section{References}

[1] M. Abramowitz, I.A. Stegun (eds.), Handbook of mathematical functions with formulas, graphs, and mathematical tables. National Bureau of Standards, Applied Mathematics Series, vol. 55. 9th printing, Dover, New York, 1972.

[2] R.P. Agarwal, H. Agarwal, S.K. Sen, Birth, growth and computation of pi to ten trillion digits, Adv. Difference Equ. 2013, 2013:100, 59 pp. http://www.advancesindifferenceequations.com/content/2013/1/100

[3] T. Amdeberhan, O. Espinosa, V.H. Moll, A. Straub, Wallis-Ramanujan-Schur-Feynman, Amer. Math. Monthly 117 (2010) 618-632.

[4] P. Beckmann, A History of Pi, St. Martin’s Press, New York, New York, 1971.

[5] L. Berggren, J. Borwein, P. Borwein (eds.), Pi: A Source Book, 2nd ed., Springer, New York, 2000.

[6] T. Buric, N. Elezović, R. Šimić, Asymptotic expansions of the multiple quotients of gamma functions with applications. Math. Inequal. Appl. 16 (2013) 1159-1170.

[7] C.-P. Chen, J. Choi, Two infinite product formulas with two parameters, Integral Transforms Spec. Funct. 24 (2013) 357-363. 
[8] C.-P. Chen, N. Elezović, L. Vukšić, Asymptotic formulae associated with the Wallis power function and digamma function, J. Class. Anal. 2 (2013) 151-166.

[9] C.-P. Chen, R.B. Paris, Generalizations of two infinite product formulas, Integral Transforms Spec. Funct. 25 (2014) 547-551.

[10] C.-P. Chen, R.B. Paris, On the asymptotics of products related to generalizations of the Wilf and Mortini problems, Integral Transforms Spec. Funct. DOI: 10.1080/10652469.2015.1118627

[11] J. Choi, J. Lee, H.M. Srivastava, A generalization of Wilf's formula, Kodai. Math. J. 26 (2003) $44-48$.

[12] J. Choi, T.Y. Seo, Identities involving series of the Riemann zeta function, Indian. J. Pure Appl. Math. 30 (1999) 649-652.

[13] J.-E. Deng, T. Ban, C.-P. Chen, Sharp inequalities and asymptotic expansion associated with the Wallis sequence, J. Ineq. Appl. 2015, 2015: 186. http://www.journalofinequalitiesandapplications.com/content/2015/1/186

[14] W. Dunham, Journey Through Genius, The Great Theorems of Mathematics, Penguin, 1990.

[15] N. Elezović, L. Lin, L. Vukšić, Inequalities and asymptotic expansions for the Wallis sequence and the sum of the Wallis ratio, J. Math. Inequal. 7 (2013) 679-695.

[16] J. Guillera, J. Sondow, Double integrals and infinite products for some classical constants via analytic continuations of Lerch's transcendent, Ramanujan J. 16 (2008) 247-270.

[17] M.D. Hirschhorn, Comments on the paper "Wallis sequence estimated through the Euler-Maclaurin formula: even from the Wallis product $\pi$ could be computed fairly accurately" by V. Lampret. Aust. Math. Soc. Gaz. 32 (2005) 194.

[18] V. Lampret, An asymptotic approximation of Wallis' sequence, Cent. Eur. J. of Math. 10 (2012) 775-787.

[19] V. Lampret, Wallis sequence estimated through the Euler-Maclaurin formula: even from the Wallis product $\pi$ could be computed fairly accurately, Austral. Math. Soc. Gaz. 31 (2004) 328-339.

[20] L. Lin, Further refinements of Gurland's formula for $\pi$, J. Ineq. Appl. 2013, 2013:48. http://www.journalofinequalitiesandapplications.com/content/2013/1/48

[21] L. Lin J.-E. Deng, C.-P. Chen, Inequalities and asymptotic expansions associated with the Wallis sequence. J. Ineq. Appl. 2014, 2014:251. http://www.journalofinequalitiesandapplications.com/content/2014/1/251.

[22] Y.L. Luke, The Special Functions and their Approximations, vol. I, Academic Press, New York, 1969.

[23] S.J. Miller, A Probabilistic Proof of Wallis's Formula for $\pi$, Amer. Math. Monthly 115 (2008) 740745.

[24] C. Mortici, Estimating $\pi$ from the Wallis sequence, Math. Commun. 17 (2012) 489-495. 
[25] C. Mortici, V.G. Cristea, D. Lu, Completely monotonic functions and inequalities associated to some ratio of gamma function, Appl. Math. Comput. 240 (2014) 168-174.

[26] C. Mortici, Completely monotone functions and the Wallis ratio, Appl. Math. Lett. 25 (2012) $717-$ 722.

[27] C. Mortici, Sharp inequalities and complete monotonicity for the Wallis ratio, Bull. Belg. Math. Soc. Simon Stevin, 17 (2010) 929-936.

[28] C. Mortici, A new method for establishing and proving new bounds for the Wallis ratio, Math. Inequal. Appl. 13 (2010) 803-815.

[29] C. Mortici, New approximation formulas for evaluating the ratio of gamma functions, Math. Comput. Modelling 52 (2010) 425-433.

[30] G. Myerson, The limiting shape of a sequence of rectangles, Amer. Math. Monthly 99 (1992) 279280.

[31] E. Păltănea, On the rate of convergence of Wallis' sequence, Austral. Math. Soc. Gaz. 34 (2007) 34-38.

[32] J. Ser, Sur une expression de la fonction $\zeta(s)$ de Riemann, C. R. Acad. Sci. Paris Sér. I Math. 182 (1926) 1075-1077.

[33] A. Sofo, Some representations of $\pi$, Austral. Math. Soc. Gaz. 31 (2004) 184-189.

[34] A. Sofo, $\pi$ and some other constants, J. Inequal. Pure Appl. Math. 6 (5) (2005), Article 138 (electronic).

[35] J. Sondow, An infinite product for $e^{\gamma}$ via hypergeometric formulas for Euler's constant $\gamma$, (2003, preprint).http://arXiv.org/abs/math/0306008.

[36] J. Sondow, A faster product for $\pi$ and a new integral for $\ln (\pi / 2)$, Amer. Math. Monthly 112 (2005) $729-734$.

[37] J. Wästlund, An Elementary Proof of the Wallis Product Formula for Pi, Amer. Math. Monthly 114 (2007) 914-917.

[38] Wikipedia contributors, Mertens' theorems, Wikipedia, The Free Encyclopedia, https://en.wikipedia.org/wiki/Mertens27_theorems.

[39] H.S. Wilf, Problem 10588, Problems and Solutions, Amer. Math. Monthly 104 (1997) 456. 